\title{
Deviations from Tri-Bimaximality and Quark-Lepton Complementarity
}

\author{
Yusuke Shimizu ${ }^{1, *}$ and Ryo Takahashi ${ }^{2, \dagger}$ \\ ${ }^{1}$ Graduate School of Science and Technology, Niigata University, \\ Niigata 950-2181, Japan \\ ${ }^{2}$ Max-Planck-Institut für Kernphysik, Saupfercheckweg 1, \\ 69117 Heidelberg, Germany
}

\begin{abstract}
We study deviations from tri-bimaximality (TBM) and quark-lepton complementarity (QLC) in a model independent way. The current neutrino experimental data is well approximated by tri-bimaximal generation mixing but the QLC relations are not satisfied with each data of $1 \sigma$ level. This means that there exist deviations from the complementarity. The same fact for the TBM might be checked in the future neutrino experiments. We discuss such deviations from the TBM and QLC, simultaneously. A new ratio between the deviations is introduced, and some interesting points are presented. We also show predicted correlations among leptonic mixing angles at the points.
\end{abstract}

\footnotetext{
*E-mail address: shimizu@muse.sc.niigata-u.ac.jp

${ }^{\dagger}$ E-mail address: ryo.takahashi@mpi-hd.mpg.de
} 


\section{Introduction}

The current precision measurements of neutrino oscillation have suggested that there are two large mixing angles among three generations in the lepton sector unlike the quark sector. It is known that the experimental data of mixing angles [1] is approximated by the tri-bimaximal generation mixing [2], which is described as

$$
V_{\mathrm{TB}}=\left(\begin{array}{ccc}
2 / \sqrt{6} & 1 / \sqrt{3} & 0 \\
-1 / \sqrt{6} & 1 / \sqrt{3} & -1 / \sqrt{2} \\
-1 / \sqrt{6} & 1 / \sqrt{3} & 1 / \sqrt{2}
\end{array}\right) .
$$

This matrix leads to the following values of mixing angles:

$$
\sin ^{2} \theta_{12}^{l}=\frac{1}{3}, \quad \sin ^{2} \theta_{23}^{l}=\frac{1}{2}, \quad \sin ^{2} \theta_{13}^{l}=0
$$

or equivalently,

$$
\theta_{12}^{l} \simeq 35.3^{\circ}, \quad \theta_{23}^{l}=45^{\circ}, \quad \theta_{13}^{l}=0
$$

where $\theta_{i j}^{l}(i, j=1,2,3 ; i<j)$ are the Pontecorvo-Maki-Nakagawa-Sakata (PMNS) [3] mixing angles. This is one of interesting theoretical suggestions, and thus, such a suggestive form of generation mixing matrix strongly motivates the search for a hidden flavour structure of the lepton sector. In fact, a number of proposals based on a flavour symmetry have been elaborated [4, 5]. The observed values of PMNS mixing angles from the current neutrino oscillation experiments [1] are

$$
\theta_{12}^{l}=\left(34.3_{-0.991}^{+1.16}\right)^{\circ}, \quad \theta_{23}^{l}=\left(45_{-3.45}^{+4.02}\right)^{\circ}, \quad \theta_{13}^{l}=\left(6.55_{-2.92}^{+2.73}\right)^{\circ},
$$

at $1 \sigma$ level. We find that there are small deviations of the best-fit values for solar and reactor angles from the TBM while the best-fit value of atmospheric angle equals the one of TBM.

Regarding generation mixing angles including the quark sector, intriguing relations among mixing angles of quark and lepton sectors have been proposed in [6, 7], which is called quarklepton complementarity (QLC). The originally proposed QLC relation is described as

$$
\theta_{12}^{l}+\theta_{12}^{q}=\frac{\pi}{4}=45^{\circ}
$$

where $\theta_{i j}^{q}$ are the Cabibbo-Kobayashi-Maskawa mixing angles [8]. The second and third QLC relations can also be written as

$$
\begin{aligned}
& \theta_{23}^{l}+\theta_{23}^{q}=\frac{\pi}{4}=45^{\circ}, \\
& \theta_{13}^{l}+\theta_{13}^{q}=0 .
\end{aligned}
$$


In ref. [6], a realization of QLC relations has been proposed in the context of the grand unified theory with non-Abelian flavour symmetry. Then implication of relations for the quark-lepton symmetry and the mechanism of neutrino mass generation has been discussed.

The current mixing angles for the quark sector [9] are given at $1 \sigma$ as

$$
\sin \theta_{12}^{q}=0.2257 \pm 0.0010, \quad \sin \theta_{23}^{q}=0.0415_{-0.0011}^{+0.0010}, \quad \sin \theta_{13}^{q}=0.00359 \pm 0.00016,
$$

or equivalently,

$$
\theta_{12}^{q}=\left(13.0_{-0.0588}^{+0.118}\right)^{\circ}, \quad \theta_{23}^{q}=\left(2.38_{-0.0631}^{+0.0573}\right)^{\circ}, \quad \theta_{13}^{q}=\left(0.206_{-0.00917}^{+0.00917}\right)^{\circ} .
$$

Therefore, we find from the current experimental data [1, 9] that the above relations (5) and (77) are not satisfied with each data of $1 \sigma$ :

$$
\theta_{12}^{l}+\theta_{12}^{q} \simeq\left(47.4_{-1.05}^{+1.21}\right)^{\circ}, \quad \theta_{13}^{l}+\theta_{13}^{q} \simeq\left(6.75_{-2.93}^{+2.74}\right)^{\circ}
$$

The second QLC relation ([6) can be satisfied with each data of $1 \sigma$ level:

$$
\theta_{23}^{l}+\theta_{23}^{q} \simeq\left(47.4_{-3.51}^{+4.08}\right)^{\circ}
$$

One of the most important missions for the neutrino oscillation experiments is to clarify whether the reactor angle is zero or not. The finiteness of the reactor angle means of course that the TBM is ruled out. The improvement of accuracy to determine the solar and atmospheric angles is also an important task. Since the QLC relations are related with the leptonic mixing angles, the correlations make us possible to investigate the TBM and QLC, simultaneously. In this letter, we focus on deviations from TBM and QLC, simultaneously, towards upcoming data from neutrino oscillation experiments.

This paper is organized as follows. In the second section, we define deviations from the TBM and QLC, and discuss the relations among the leptonic mixing angles and magnitudes of deviations while focusing on the current experimental bounds and future sensitivity for measuring the mixing angle of the reactor neutrino. Next we introduce a new ratio between the deviations from TBM and QLC, and show a relation of leptonic mixing angles with the introduced ratio. We also point out four relatively interesting points of this ratio. Then correlations of three leptonic mixing angles are shown in these points. The third section is devoted to the summary of our results.

\section{Deviations from tri-bimaximality and quark-lepton complementarity}

First, we define deviations from the TBM and QLC as

$$
\begin{aligned}
\delta_{\mathrm{TBM}} & \equiv \sum_{i<j}\left[\theta_{i j}^{l}-\theta_{i j}^{\mathrm{TBM}}\right] \\
\delta_{\mathrm{QLC}} & \equiv \sum_{i<j}\left[\left(\theta_{i j}^{l}+\theta_{i j}^{q}\right)-\theta_{i j}^{\mathrm{QLC}}\right]
\end{aligned}
$$


(a)

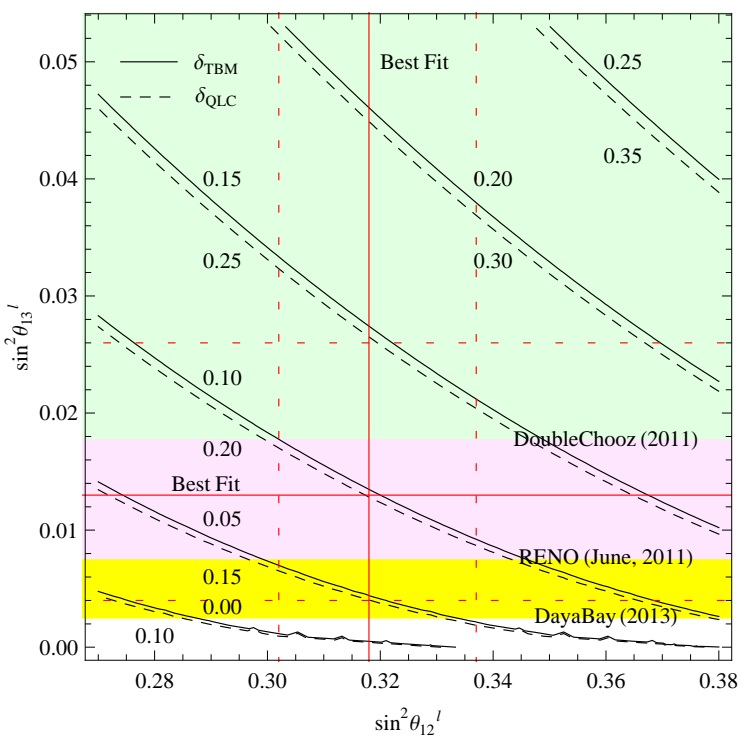

(b)

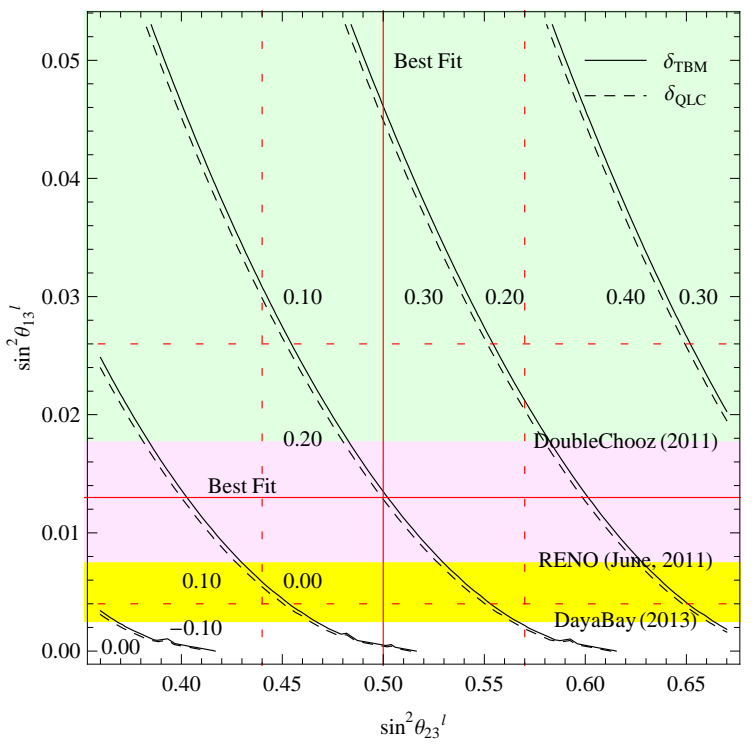

Figure 1: Deviations from the TBM and QLC.

respectively, where the mixing angles $\theta_{i j}^{l, q}$ are experimentally observed values, and $\theta_{12}^{\mathrm{QLC}}=$ $\theta_{23}^{\mathrm{QLC}}=45^{\circ}, \theta_{13}^{\mathrm{QLC}}=0$, and the values of $\theta_{i j}^{\mathrm{TBM}}$ are given in (3) 1 Figure 1 shows the contour plots of these deviations in the plane of leptonic mixing angles. Each figure is drawn within $3 \sigma$ level for each mixing angle, and the contours are given in radian units. The solid and dashed lines correspond to the values of best fit and $1 \sigma$ range, respectively. The best-fit values of atmospheric and solar angles are utilized for the figs. 1(a) and (b), respectively, and the best-fit values of all CKM mixing angles are taken in both figures. The solid and dashed curves denote the deviations from the TBM and QLC defined in (12) and (13), respectively. These figures can give clear comparisons and understandings for the deviations from the TBM and QLC. It is found that the magnitude of deviation from the QLC is larger than that from the TBM. For references, expected upper limits at 90\% CL for $\theta_{13}^{l}$, which are achieved by the DoubleChooz [11], RENO [12], and DayaBay [13] experiments one after another, are also shown by coloured regions. The times given in brackets for each experiment are roughly estimated by the values of $\sin ^{2} 2 \theta_{13}^{l}=0.07,0.03$, and 0.01 for the DoubleChooz, RENO, and DayaBay experiments, respectively. Especially for the DayaBay experiment, the time is estimated from the expectation with the strongest sensitivity assumption 2

Next, let us introduce a new ratio between the deviations from TBM and QLC towards

${ }^{1}$ The discussions of experimentally observed values in the previous section are based on the PDG format of the Schechter-Valle parametrization [9, 10. However, since the parameters indicating deviations (12) and (13) are defined by just mixing angles, the results given in this letter do not depend on the parametrization for the CKM and PMNS mixing matrices. We also discuss in ranges of $0 \leq \theta_{i j} \leq \pi / 2$ for all mixing angles throughout of this letter.

${ }^{2}$ See 14 for a recent excellent review about the present status and prospect of $\theta_{13}^{l}$ measurements. 
a more profound understanding of deviations as

$$
R \equiv \frac{\left|\delta_{\mathrm{TBM}}\right|}{\delta_{\mathrm{QLC}}} .
$$

This ratio can take a finite positive value or zero because of the positive value of $\delta_{\mathrm{QLC}}$ with each data of $1 \sigma$ level. The ratio becomes zero at the tri-bimaximal limit. Figure 2 shows this ratio as a function of the sum of leptonic mixing angles, $\sum \theta_{i j}^{l}$. The best-fit values of all CKM mixing angles have been taken. Since the deviation from the TBM, $\delta_{\mathrm{TBM}}$, can become negative, the function of this ratio forms like a valley. The tri-bimaximal mixing corresponds to the bottom of the valley. We also show expected upper limits at 90\% CL for $\theta_{13}^{l}$, which might be achieved by the DoubleChooz, RENO, and DayaBay experiments with the maximal values for both $\theta_{12}^{l}$ and $\theta_{23}^{l}$ at $1 \sigma$ level in fig. 2(a), with the best-fit values for the ones in fig. 2(b), and with the minimal values in fig. 2(c). These show relations among the ratio $R$ and the sum of the leptonic mixing angles, and future sensitivities for the measurement of $\theta_{13}^{l}$ in the upcoming neutrino oscillation experiments.

It might be the most suggestive case for particle physics if the exact tri-bimaximal mixing (bottom of the valley) could be satisfied in Nature. In this letter, we point out other suggestive scenarios in terms of deviations from the TBM and QLC. They are labelled by $R_{1}, R_{ \pm 0.5}$, and $R_{0}$ in fig. 2. We call those points as even, half , and cancelling deviation scenarios, respectively, and the values of the ratio at these points are as follows:

even deviation

$$
R_{1}: R=1 \quad \text { and } \quad \delta_{\mathrm{TBM}}=-\delta_{\mathrm{QLC}},
$$

half- deviation

$$
R_{-0.5}: \quad R=0.5 \text { and } \delta_{\mathrm{TBM}}=-\frac{1}{2} \delta_{\mathrm{QLC}}
$$

half + deviation

$$
R_{+0.5}: \quad R=0.5 \text { and } \delta_{\mathrm{TBM}}=+\frac{1}{2} \delta_{\mathrm{QLC}}
$$

canceling deviation

$$
R_{0}: R=0 \text { and } \delta_{\mathrm{TBM}}=0
$$

Notice that the exact tri-bimaximal mixing corresponds to the point labeled by $R_{0}$ but this point does not necessarily mean only the exact tri-bimaximal mixing, that is, that includes cancelled solutions among the deviation from TBM (see (12) for the definition of deviation). The even deviation scenario shown by $R_{1}$ means that the absolute value of deviation from TBM equals that from the QLC, one can achieve this scenario with leptonic mixing angles at $3 \sigma$ range. This point can be realized by a negative value of $\delta_{\mathrm{TBM}}$. Finally, half deviation 
(a)

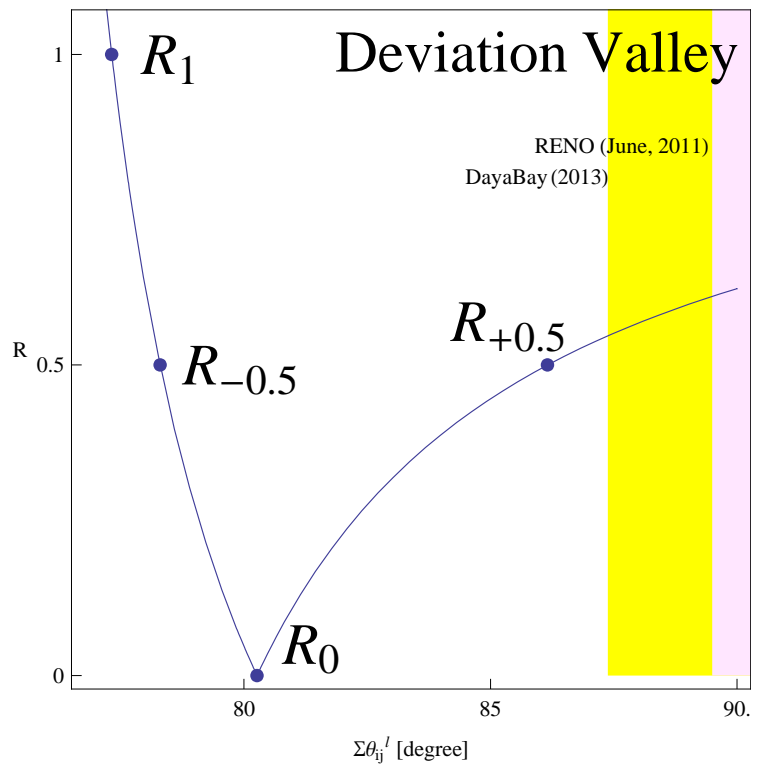

(b)

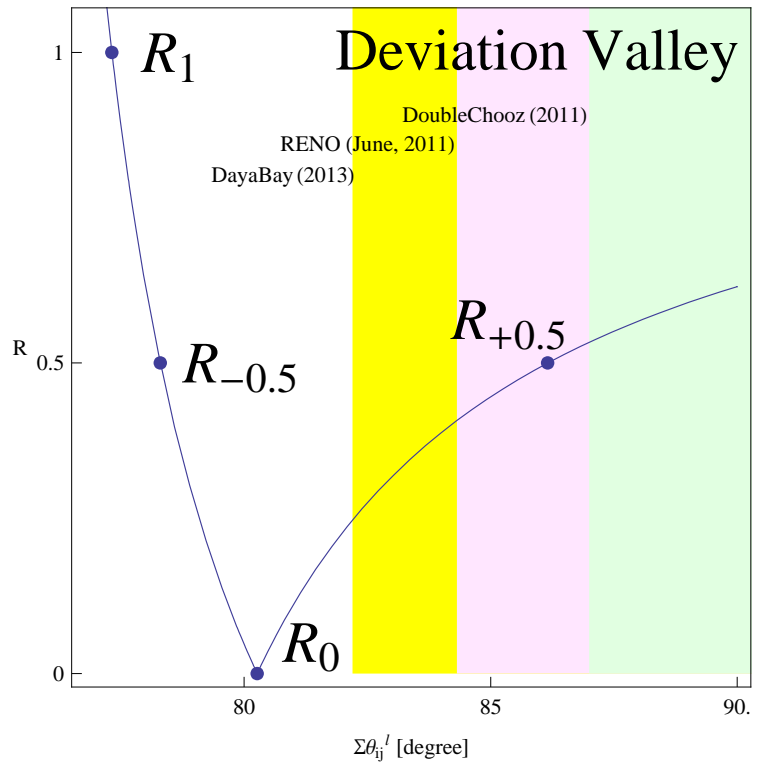

(c)

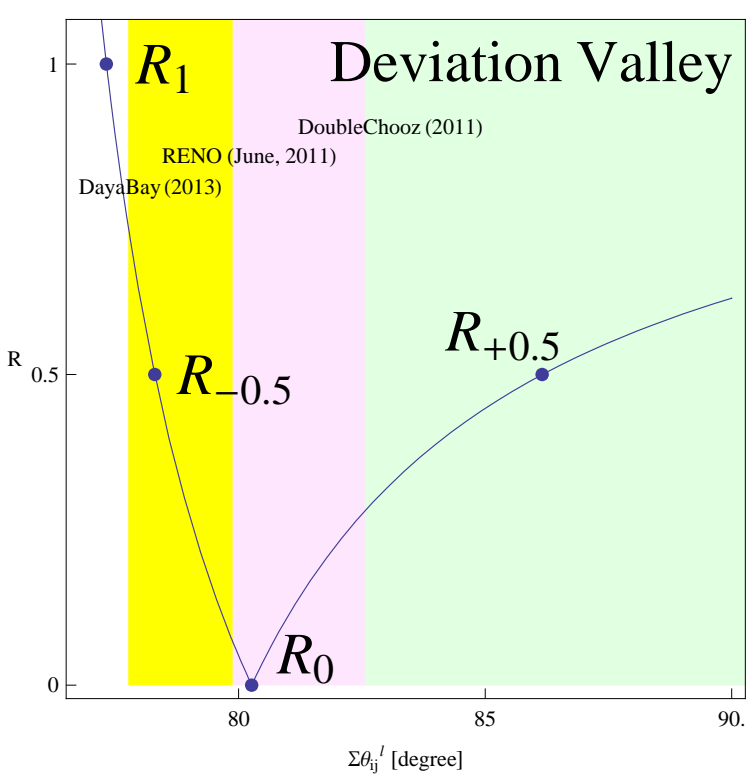

Figure 2: Ratio between deviations from TBM and QLC. 


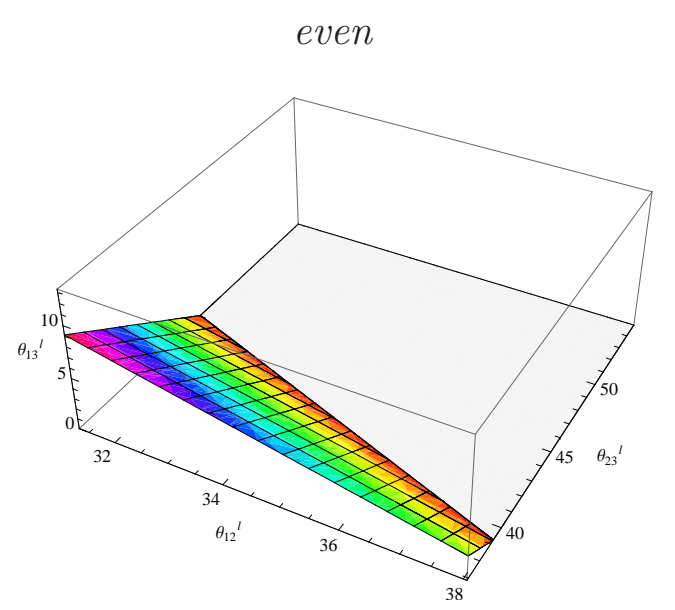

half

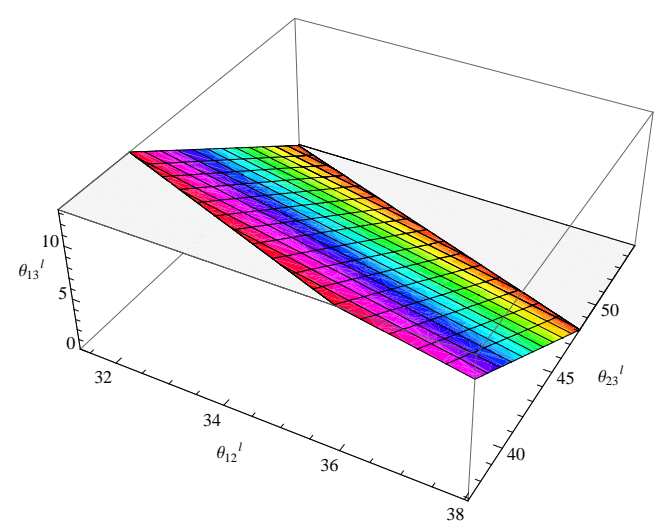

half

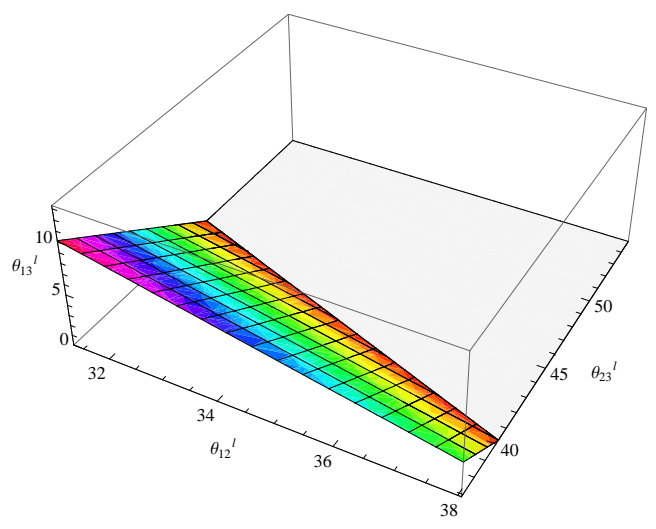

cancelling

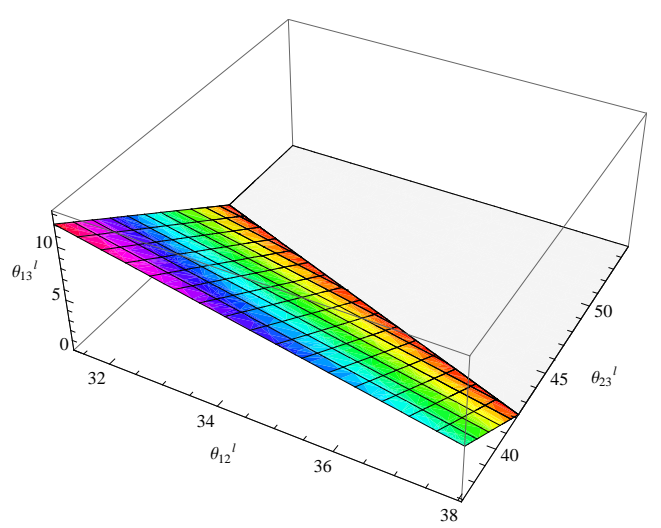

Figure 3: Predicted surfaces of leptonic mixing angles from four scenarios.

scenarios shown by $R_{ \pm 0.5}$ denote that the magnitude of deviation from TBM becomes the half of that from QLC, whose point can be obtained from both positive and negative $\delta_{\text {TBM }}$. The half $_{+}$and half_ deviation scenarios are distinguished by the positive and negatives values of $\delta_{\text {TBM }}$, respectively. Notice that once the value of $R$ and the sign of $\delta_{\text {TBM }}$ are fixed we can find a unique surface in the space of leptonic mixing angles. The predicted surfaces corresponding to each deviation scenario are shown in fig. 3. It is easily seen that the value of the reactor mixing angle for fixed values of atmospheric and solar mixing angles becomes larger as we proceed from $R_{1}$ to $R_{+0.5}$ through the valley.

All flavour models to discuss quark/lepton mixing are on the curve shown in fig. 2. It is worth studying classification of all flavour models and constructing a model, which realizes suggestive scenarios based on this point of view about the ratio between deviations. In our new direction of simultaneous discussion about deviations from the TBM and QLC, it might be still very interesting if experimentally determined leptonic mixing angles could be somewhere in surfaces shown in fig. [3 except for the exact tri-bimaximal point, $\sin ^{2} \theta_{12}=1 / 3$, $\sin ^{2} \theta_{23}=1 / 2$, and $\sin \theta_{13}=0$. Without our new direction, one would argue about deviations from the TBM and QLC for each mixing angle, independently. However, once we introduce 
deviation parameters, $\delta_{\mathrm{TBM}}, \delta_{\mathrm{QLC}}$, and $R$, leptonic mixing angles somewhere in surfaces of fig. 3 can suggest even, half , or canceling deviation scenario. That will strongly motivate the construction of flavour models which should clarify a new physics (mechanism) behind the deviations from TBM and QLC in addition to investigation of the origin of TBM and QLC. For instance, if the observed solar and reactor angles are in the canceling scenario with maximal atmospheric angle, that means that the magnitude of deviation of the solar angle from the tri-bimaximal solar mixing is just the same as the size of the reactor angle;

$$
\sin ^{2}\left(\theta_{12}^{l}+\theta_{13}^{l}\right)=\frac{1}{3}
$$

when $\sin ^{2} \theta_{23}^{l}=1 / 2$. We note that this example (19) shows a correlation between solar and reactor angles, which includes the exact tri-bimaximal mixing angles as the most suggestive point. Such relatively model-dependent studies based on our proposal and further discussions about resultant predictions will be presented in separate publications.

Finally, we show an estimation of the ratio $R$ in the $A_{4}$ model [5] as an example. In ref. [5], the ratio is calculated as $R=0$ since the model can predict the exact tri-bimaximal mixing at the leading order. However, the next-to-leading order (NLO) corrections give deviations from the TBM [15]. Typical values of leptonic mixing angles up to the NLO are estimated as

$$
\sin ^{2} \theta_{12}=0.36, \quad \sin ^{2} \theta_{13}=4.8 \times 10^{-6}, \quad \sin ^{2} \theta_{23}=0.48 .
$$

Therefore, the value of the ratio becomes $R \simeq 0.085 \simeq \mathcal{O}(0.1)$, where the best-fit values of all CKM mixing angles are taken.

\section{Summary}

In this letter, we have studied deviations from TBM and QLC in a model-independent way. First, we have defined those deviations, and then, presented those contours while comparing with upcoming reactor neutrino experiments. Once we fixed the best-fit value of the solar or atmospheric angle, the deviation from QLC is larger than that from TBM. Next, a new ratio between deviations from TBM and QLC has been introduced. We have focused on the ratio, and pointed out relatively suggestive four scenarios, which were named as even, half $f_{ \pm}$and canceling deviation scenarios. Each scenario can predict a different surface with a correlation among the three leptonic mixing angles. If the future neutrino oscillation experiments could suggest that observed values of mixing angles are somewhere in the above scenario, our new proposal to understand deviations from TBM and QLC would strongly motivate the search for further hidden flavour structures of the standard model in addition to a clarification of the origins of TBM and QLC. It might be worth recognizing that some exotic correlations among mixing angles can be predicted in relatively model-dependent ways.

\section{Acknowledgements}

We would like to thank M. Lindner and M. Tanimoto for helpful comments. The work of Y.S. is supported by the Japan Society of Promotion of Science. The work of R.T. is supported 
by the DFG-SFB TR 27. Y.S. is grateful to Max-Planck-Institut für Kernphysik for their hospitality.

\section{References}

[1] T. Schwetz, M. Tortola and J. W. F. Valle, New J. Phys. 10 (2008) 113011.

[2] P. F. Harrison, D. H. Perkins and W. G. Scott, Phys. Lett. B 530 (2002) 167; P. F. Harrison and W. G. Scott, Phys. Lett. B 535 (2002) 163;

[3] Z. Maki, M. Nakagawa and S. Sakata, Prog. Theor. Phys. 28 (1962) 870; B. Pontecorvo, Sov. Phys. JETP 26 (1968) 984 [Zh. Eksp. Teor. Fiz. 53 (1967) 1717].

[4] E. Ma, Phys. Rev. D 70 (2004) 031901; S. F. King, JHEP 0508 (2005) 105; I. de Medeiros Varzielas and G. G. Ross, Nucl. Phys. B 733 (2006) 31; K. S. Babu and X. G. He, arXiv:hep-ph/0507217. E. Ma, Phys. Lett. B 632 (2006) 352; A. Zee, Phys. Lett. B 630 (2005) 58; W. Grimus and L. Lavoura, JHEP 0601 (2006) 018; E. Ma, Phys. Rev. D 73 (2006) 057304; G. Altarelli and F. Feruglio, Nucl. Phys. B 741 (2006) 215; J. E. Kim and J. C. Park, JHEP 0605 (2006) 017; I. de Medeiros Varzielas, S. F. King and G. G. Ross, Phys. Lett. B 644 (2007) 153; R. N. Mohapatra, S. Nasri and H. B. Yu, Phys. Lett. B 639 (2006) 318; I. de Medeiros Varzielas, S. F. King and G. G. Ross, Phys. Lett. B 648 (2007) 201; E. Ma, Mod. Phys. Lett. A 21 (2006) 2931; G. Altarelli, F. Feruglio and Y. Lin, Nucl. Phys. B 775 (2007) 31; H. Zhang, Phys. Lett. B 655 (2007) 132; P. D. Carr and P. H. Frampton, arXiv:hep-ph/0701034; F. Feruglio, C. Hagedorn, Y. Lin and L. Merlo, Nucl. Phys. B 775 (2007) 120 [Erratum-ibid. 836 (2010) 127]; M. C. Chen and K. T. Mahanthappa, Phys. Lett. B 652 (2007) 34; C. Luhn, S. Nasri and P. Ramond, Phys. Lett. B 652 (2007) 27; Y. Koide, arXiv:0707.0899 [hep-ph]; E. Ma, Phys. Lett. B 660 (2008) 505; F. Bazzocchi, S. Morisi and M. Picariello, Phys. Lett. B 659 (2008) 628; F. Plentinger, G. Seidl and W. Winter, JHEP 0804 (2008) 077; F. Plentinger and G. Seidl, Phys. Rev. D 78 (2008) 045004; S. Antusch, S. F. King and M. Malinsky, JHEP 0805 (2008) 066; Y. Lin, Nucl. Phys. B 813 (2009) 91; N. Haba, R. Takahashi, M. Tanimoto and K. Yoshioka, Phys. Rev. D 78 (2008) 113002; F. Feruglio, C. Hagedorn, Y. Lin and L. Merlo, Nucl. Phys. B 809 (2009) 218; T. Araki and R. Takahashi, Eur. Phys. J. C 63 (2009) 521; W. Grimus and L. Lavoura, JHEP 0904 (2009) 013; H. Ishimori, Y. Shimizu and M. Tanimoto, Prog. Theor. Phys. 121 (2009) 769; S. Morisi, Phys. Rev. D 79 (2009) 033008; F. Bazzocchi, L. Merlo and S. Morisi, Nucl. Phys. B 816 (2009) 204; F. Bazzocchi, L. Merlo and S. Morisi, Phys. Rev. D 80 (2009) 053003; G. Altarelli and D. Meloni, J. Phys. G 36 (2009) 085005; M. Hirsch, S. Morisi and J. W. F. Valle, Phys. Lett. B 679 (2009) 454; Y. Lin, Nucl. Phys. B 824 (2010) 95; D. Aristizabal Sierra, F. Bazzocchi, I. de Medeiros Varzielas, L. Merlo and S. Morisi, Nucl. Phys. B 827 (2010) 34; F. Feruglio, C. Hagedorn and L. Merlo, JHEP 1003 (2010) 084; Y. Lin, L. Merlo and A. Paris, Nucl. Phys. B 835 (2010) 238; F. Feruglio, C. Hagedorn, Y. Lin and L. Merlo, arXiv:0911.3874 [hep-ph]; H. Ishimori, K. Saga, Y. Shimizu and M. Tanimoto, Phys. Rev. D 81 (2010) 115009. 
[5] G. Altarelli and F. Feruglio, Nucl. Phys. B 720 (2005) 64.

[6] M. Raidal, Phys. Rev. Lett. 93 (2004) 161801.

[7] H. Minakata and A. Y. Smirnov, Phys. Rev. D 70 (2004) 073009.

[8] N. Cabibbo, Phys. Rev. Lett. 10 (1963) 531; M. Kobayashi and T. Maskawa, Prog. Theor. Phys. 49 (1973) 652.

[9] C. Amsler et al. [Particle Data Group], Phys. Lett. B 667 (2008) 1.

[10] J. Schechter and J. W. F. Valle, Phys. Rev. D 22 (1980) 2227.

[11] F. Ardellier et al. [Double Chooz Collaboration], arXiv:hep-ex/0606025.

[12] J. K. Ahn [RENO Collaboration], arXiv:1003.1391 [hep-ex].

[13] X. Guo et al. [Daya-Bay Collaboration], arXiv:hep-ex/0701029.

[14] M. Mezzetto and T. Schwetz, arXiv:1003.5800 [hep-ph].

[15] A. Hayakawa, H. Ishimori, Y. Shimizu and M. Tanimoto, Phys. Lett. B 680 (2009) 334. 